\title{
The First Example of an Intramolecular Westphal Reaction. Synthesis of a New Aza-Quinolizinium Type System.
}

\author{
Valentín Martínez-Barrasa, Carolina Burgos, M. Luisa Izquierdo \\ Julio Alvarez-Builla and Juan J. Vaquero*
}

Departamento de Química Orgánica. Universidad de Alcalá.

28871 Alcalá de Henares. Madrid. Spain.

Received 12 February 1999; accepted 6 April 1999

\begin{abstract}
The first example of an intramolecular Westphal condensation is described. To test the utility of this reaction, new benz $(f)$ azino $[2,1-a]$ phthalazinium salts have been prepared from appropriate dicarbonyl precursors. ○ 1999 Elsevier Science Ltd. All rights reserved.

Keywords: Westphal; condensations; quinolizinium ions; phthalazines.
\end{abstract}

In a current research project focused on the synthesis and biochemistry of novel DNA intercalators based on quinolizinium and aza-quinolizinium chromophores [1-4], the Westphal reaction [5] has proved to be a useful tool for preparing these polycyclic systems containing a bridgehead quaternary nitrogen. Although the procedure has been described for C-C [5-7], N-N [8-11] and N-C [12-15] substrates, it had always being used in its intermolecular version.
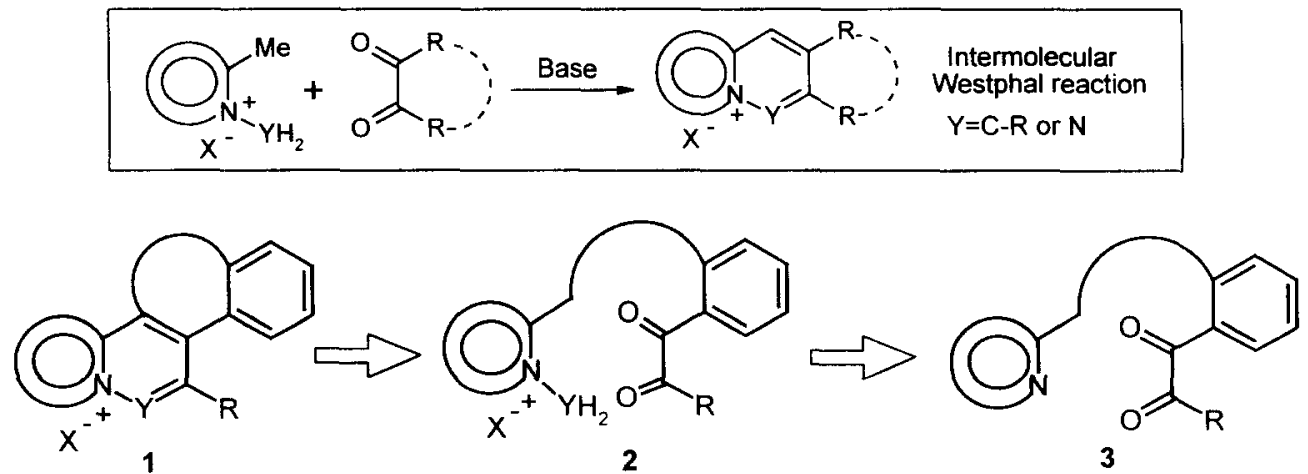

Scheme 1: Westphal reaction and retrosynthetic analysis of the aza-quinolizinium cation 1 
In an attempt to extend this simple and straightforward methodology to the preparation of new aza-quinolizinium systems, we decided to explore the viability of the intramolecular process and here we communicate the first successful example, applied to the synthesis of the benz(f)azino[2,1-a]phthalazinium system 1, a new cationic tetracyclic system not available by the classical intermolecular process. The aza-quinolizinium cation $\mathbf{1}$ is a benzo-analogue of the known pyrido[2,1-a]phthalazinium ion, which has been recently prepared [16] by acid catalysed ring closure of 1-amino-2-(2'-[1,3]dioxolan-2-yl-phenyl)pyridinium salt. The parent compound of the latter, pyrido[1,2-b]pyridazinium has been previously described [12]. The retrosynthesis of 1 is outlined in Scheme 1 and involves the intramolecular condensation of the aminated salt 2 , with the diketone 3 being the key intermediate.
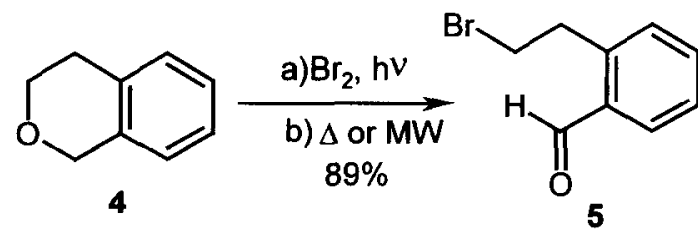<smiles>[R]C=Cc1ccccc1CCBr</smiles>

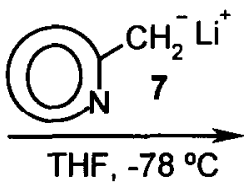

$62-98 \%$<smiles>[R]C=Cc1ccccc1CCCc1ccccn1</smiles>

8

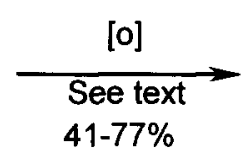

$41-77 \%$<smiles>[R]C(=O)C(=O)c1ccccc1CCCc1ccccn1</smiles>

3

Scheme 2: Preparation of dicarbonyl compounds 3.

Scheme 2 displays the preparation of the diketones 3 , starting from isochroman 4 . Bromination of commercially available 4 in the presence of sunlight, followed by thermolysis gives 5 [17,18], but better results were obtained during the course of this work by microwave irradiation ( 1 min at 362 watts) of the bromination product of 4 adsorbed on silica gel [19]. Wittig olefination of 5 was carried out by treatment with the corresponding phosphonium salt and $n$-BuLi, (THF, $-78^{\circ} \mathrm{C}$ ) to yield a cis/trans mixture of alkenes 6 . Nucleophilic displacement of bromide in 6, by $\alpha$-azinylmethylides 7 [20] produced 8 in excellent yield.

The 1,2-dicarbonyl system in $3(\mathrm{R}=\mathrm{Ar})$ was then smoothly generated by oxidation of the styryl moiety in 8 using various DMSO-reagents ( $\mathrm{HBr} / \mathrm{DMSO}, \mathrm{I}_{2} / \mathrm{DMSO}$ and $\mathrm{PdCl}_{2} / \mathrm{DMSO}$ ) [21-24]'. Other non DMSO-based oxidative systems, such as Katritzky's reagent [25], were

'These methods can be successfully applied when $R$ is an aromatic substituent, providing the corresponding 1,2-diketones 3 under mild conditions. However they are not suitable reagents for preparative oxidation of alkyl olefins when arylcarboxylic acids and aldehydes were found in the main reaction products. 
also tried, although they did not provide satisfactory results.

The preparation of the $\mathrm{N}$-aminoazinium salts 2 was performed by direct amination of the dicarbonylazine compounds 3 , using (o-mesitylenesulfonyl)hydroxylamine ( $\mathrm{MSH}$ ), in $\mathrm{CH}_{2} \mathrm{Cl}_{2}$ at room temperature [13,26] (Scheme 3). Having the substrates in hand, we performed the intramolecular condensation using different bases and solvents, with sodium acetate/acetone [12] and triethylamine/ethanol [1], giving the best yields. ${ }^{2}$ Results are summarised in Table 1.

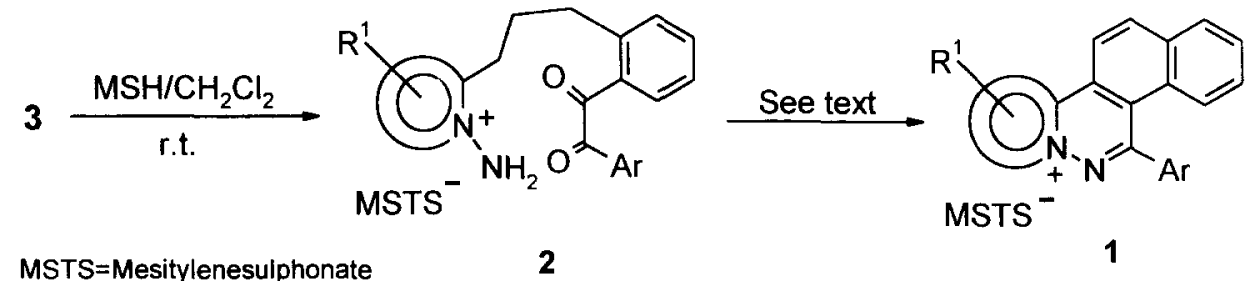

Scheme 3: Preparation of compounds 2 and 1.

Table 1

Benz(f)azino[2,1-a]phthalazinium salts 1 prepared by intramolecular Westphal reactions.

\begin{tabular}{|c|c|c|c|c|c|}
\hline \multirow[t]{2}{*}{ Entry } & \multirow[t]{2}{*}{ Azine } & \multirow[t]{2}{*}{$\mathrm{R}^{\prime}$} & \multirow[t]{2}{*}{$\overline{A r}$} & \multicolumn{2}{|c|}{ Yield $(\%)^{\mathrm{a}}$} \\
\hline & & & & 2 & 1 \\
\hline 1 & & $\mathbf{H}$ & $\mathrm{C}_{6} \mathbf{H}_{5}$ & 92 & $65^{\mathrm{b}}$ \\
\hline 2 & & 4-Me & $\mathrm{C}_{6} \mathrm{H}_{5}$ & 76 & $28^{b}$ \\
\hline 3 & & 6-Me & $\mathrm{C}_{6} \mathrm{H}_{5}$ & 83 & $50^{c}$ \\
\hline 4 & & 6-Me & 4-EtO- $\mathrm{C}_{6} \mathrm{H}_{4}$ & 92 & $28^{c}$ \\
\hline 5 & & $\mathrm{H}$ & $\mathrm{C}_{6} \mathrm{H}_{5}$ & 92 & $75^{b}$ \\
\hline 6 & & $\mathrm{H}$ & 4-EtO- $\mathrm{C}_{6} \mathrm{H}_{4}$ & 75 & $29^{\mathrm{c}}$ \\
\hline 7 & & $6-\mathrm{Me}$ & $\mathrm{C}_{6} \mathrm{H}_{5}$ & 95 & $50^{\mathrm{b}}$ \\
\hline 8 & & 6-Me & 4-EtO- $\mathrm{C}_{6} \mathrm{H}_{4}$ & 84 & $50^{\mathrm{b}}$ \\
\hline
\end{tabular}

${ }^{a} Y$ ields refer to isolated pure products. All products were identified by spectroscopic and/or literature data

${ }^{\mathrm{b}}$ Sodium acetate/acetone, reflux.

${ }^{\mathrm{c}} \mathrm{Et}_{3} \mathrm{~N} / \mathrm{EtOH}$, reflux.

\footnotetext{
${ }^{2}$ Typical experimental procedure: Sodium acetate $(0.075 \mathrm{~g}, 0.91 \mathrm{mmol})$ was added to a solution of $2(0.91 \mathrm{mmol})$ in acetone (20 mL) and the mixture was heated at reflux for $6 \mathrm{~h}$. The solvent was evaporated under vacuum and the residue washed with $\mathrm{AcOEt}$ and $\mathrm{Et}_{2} \mathrm{O}$ to give 1 (Azine= pyridine, $\mathrm{R}^{\prime}=\mathrm{H}, \mathrm{Ar}=\mathrm{C}_{6} \mathrm{H}_{5}$ ) as a yellow solid, which was crystallised from $\mathrm{EtOH} / \mathrm{Et}_{2} \mathrm{O}(\mathrm{Yield}$ : $65 \%$, m.p. $=140$ $\left.142^{\circ} \mathrm{C}\right) . \delta_{\mathrm{H}}\left(300 \mathrm{MHz}, \mathrm{CD}_{3} \mathrm{OD}\right) 9.59(\mathrm{~d}, J 6.9 \mathrm{~Hz}, 1 \mathrm{H}), 9.51(\mathrm{~d}, J 9.5 \mathrm{~Hz}, 1 \mathrm{H}), 9.04(\mathrm{~d}, J 9.1 \mathrm{~Hz}, 1 \mathrm{H}), 8.78(\mathrm{t}, J 9.1 \mathrm{~Hz}, 2 \mathrm{H}), 8.30(\mathrm{t}, J 7.3$ $\mathrm{Hz} 1 \mathrm{H}), 8.26(\mathrm{~d}, J 9.1 \mathrm{~Hz}, 1 \mathrm{H}), 7.85-7.63(\mathrm{~m}, 7 \mathrm{H}), 7.44(\mathrm{t}, J 6.9 \mathrm{~Hz}, 1 \mathrm{H}), 6.79(\mathrm{~s}, 2 \mathrm{H}), 2.60(\mathrm{~s}, 6 \mathrm{H}), 2.23(\mathrm{~s}, 3 \mathrm{H})$. Analysis: for $\mathrm{C}_{3}, \mathrm{H}_{26} \mathrm{~N}_{2} \mathrm{SO}_{3}$ calculated $\mathrm{C}$ 73.49, $\mathrm{H} \mathrm{5.17, \textrm {N }} 5.53$; found C 73.20, H 4.98, N 5.78 .
} 
In conclusion benz $(f)$ azino[2,1-a]phthalazinium derivatives 1 can be obtained through a six-step sequence, using as the key step the first example of an intramolecular Westphal condensation. The methodology is currently being extended to the preparation of other related systems and results will be described in relation with their DNA intercalating properties.

Acknowledgments. The authors wish to thank the Comision Interministerial de Ciencia y Tecnología (C.I.C.Y.T.) (Project SAF98-0093) for financial support and one studentship (V. M. B.)

\section{References}

[1] Santiesteban I, Siro JG, Vaquero JJ, García-Navío JL, Alvarez-Builla J, Castaf́o O, Andrés JL. J. Org. Chem. 1995;60:56675672.

[2] Molina A, Vaquero JJ, García-Navío JL, Alvarez-Builla J, Pascual-Teresa B, Gago F, Rodrigo MM, Ballesteros M. J. Org. Chem.1996;61:5587-5599.

[3] Pastor J, Siro JG, Garcia-Navio JL, Vaquero JJ, Gago F, Pascual-Teresa B, Pastor M, Rodrigo MM. J. Org. Chem. 1997;62:5476-5483.

[4] Molina A, Vaquero JJ, García-Navío JL, Alvarez-Builla J, Pascual-Teresa B, Gago F, Rodrigo MM. J. Org. Chem. (in press)

[5] Westphal O, Jahn K, Heffe W. Arch. Pharm. 1961;294:37-45.

[6] Alvarez-Builla J, González G, Ezquerra J, Fombella ME. J. Heterocyclic Chem. 1985;22:681-685

[7] Ezquerra J, Alvarez-Builla J. J. Heterocyclic Chem. 1985;22:1151-1157.

[8] Baranova NV, Sheinkman AK, Kost AN. Khim. Geterotsikl. Soedin. 1970;8:1148.

[9] Baranova NV, Sheinkman AK, Kost AN. Khim. Geterotsikl. Soedin. 1973;9:1266-1270.

[10] Hajós G, Messmer A, Bátori S, Riedl Z. Bull. Soc. Chim. Belg. 1992;101:597-607.

[1 1] Hajós G, Riedl Z, Gács-Baitz E, Messmer A. Tetrahedron 1992;48:8459-8464.

[12] Matia MP, García-Navío JL, Vaquero JJ, Alvarez-Builla J. J. Heterocyclic Chem. 1990;27:66 1-665.

[13] Matía MP, Garcia-Navío JL, Vaquero JJ, Alvarez-Builla J. Liebigs. Ann. Chem. 1992;777-779

[14] Matia MP, Ezquerra J, García-Navío JL, Vaquero JJ, Alvarez-Builla J. Tetrahedron Lett. 1991;32:7575-7578.

[15] Molina A, García-Navío JL. Vaquero JJ, Alvarez-Builla J, Rodrigo MM, Castaño O, Andrés JL. Bioorga. Chem. Lett. 1996;6:1453-1456

[16] Messmer A, Timári GJ. Heterocyclic Chem. 1992;29:1049-1052.

[17] Yamato M, Hashigaki K, Qais N, Ishikawa S. Tetrahedron 1990;46:5909-5920.

[18] Rieche A, Schmitz E. Chem. Ber. 1956;89:1254-1262.

[19] Siro JG, Martin J, García-Navio JL, Remuiñán MJ, Vaquero JJ. Synlett. 1998;147-148.

[20] Levine R, Dimmig DA, Kadunce WM. J. Org. Chem., 1974;39:3834-3836.

[21] Yusubov MS, Filimonov VD, Ogorodnikov VD. Lzv. Akad. Nauk SSSR, Ser. Khi. 1991;868-873.

[22] Yusubov MS, Filimonov VD. Synthesis 1991;131-132.

[23] Chi KW, Yusubov MS, Filimonov VD. Synth. Commun. 1994;24:2119-2122.

[24] Yusubov MS, Filimonov VD, Vasilyeva VP, Chi KW. Synthesis 1995;1234-1236.

[25] Katritzky AR, Wang Z, Lang H, Feng D. J. Org. Chem. 1997;62:4125-4130.

[26] Tamura Y, Minamikawa J, Miki Y, Matsugashita S, Ikeda M. Tetrahedron Lett. 1972;4133-4135. 